Maria Filipowicz-Rudek

Centro de Estudos Galegos Uniwersytet Jagielloński, Kraków maria.filipowicz-rudek@uj.edu.pl

\title{
Centro de Estudos Galegos en Cracovia - bases y logros, experiencias y perspectivas
}

Keywords: Galician language, Galician culture, Centro de Estudos Galegos, cultural promotion, minority languages and cultures

Słowa kluczowe: język galicyjski, kultura galicyjska, Centro de Estudos Galegos, promocja kultury, języka i kultury mniejszości

Acaba de cumplirse el duodécimo aniversario de la fundación del Centro de Estudos Galegos en la Universidad Cracoviana. Con este motivo merece la pena mencionar los primeros momentos de la presencia del gallego en las universidades polacas, los mayores logros del Centro y las perspectivas para el futuro, tanto en el campo de la didáctica de la lengua, como en el ámbito de la promoción de la literatura y cultura gallegas. El primer Centro de Estudos Galegos en Polonia fue fundado en la Universidad de Varsovia, en el año 1997, contando siempre con un profesor-becario procedente de Galicia. Tres años más tarde, gracias a la directora de aquel entonces del Departamento de Hispánicas, la profesora Teresa Eminowicz, en el año 
2000 se inauguró en la Universidad Jaguelónica el otro centro polaco, el que tengo el honor y el gusto de coordinar desde el principio y donde llevo a cabo todas las actividades didácticas y las de difusión. Los dos centros polacos, a pesar de que funcionan en cierta medida de manera diferente, tienen como objetivo cooperar estrechamente tanto en cuanto a las actividades de promoción (conferencias, conciertos, simposios, espectáculos de cuentacuentos) como en la consecuente construcción de las bases cognitivas de la cultura gallega en Polonia en su sentido más amplio. Y lo que parece muy importante, intentar hacer arraigar estas bases en la cultura polaca.

\section{Bases y logros}

La oferta educativa en Cracovia desde el principio sigue siendo la siguiente: Lingua galega I (Nivel Inicial, A2) y Lingua galega II (Nivel Medio, B1). Así que los alumnos tienen la posibilidad de frecuentar las aulas de gallego hasta dos años, pudiendo, en casos particulares, alcanzar un nivel superior en el conocimiento del idioma gallego, cuando las clases en Cracovia van compaginadas con los cursos de verano de lengua y cultura gallegas en Galicia. La mayoría de los alumnos conoce el castellano o el portugués, o los dos idiomas. Los grupos no suelen ser muy numerosos (entre 5 y 8 alumnos), pero los que escogen las clases de gallego como materia optativa lo emprenden bastante bien motivados para seguir aprendiendo. Con vistas a ampliar un poco el perfil de los estudiantes, es decir, para poder formar también a los alumnos sin conocimiento previo de alguno de los idiomas de la Península Ibérica (en cuanto a la demanda, sí que la hay), se ha preparado un manual encaminado especialmente hacia este fin. Y aunque ya funcionaba desde hace algún tiempo como material didáctico en las aulas, en el año 2011 se publicó en forma de libro en la Editorial Księgarnia Akademicka, bajo el título A gaita galega. Manual para afondar na lingua galega. Dicho manual (escrito en coautoría con 
Lucía Rodríguez Caeiro, lectora de gallego en el Centro de Varsovia en los años 2001-2006), a diferencia de otros manuales del idioma gallego que se usan en las aulas, incluye el material gramatical, fonético y léxico organizado desde la perspectiva de un polacohablante, proporciona explicaciones detalladas de los fenómenos gramaticales inexistentes en el sistema lingüístico polaco y emplea la lengua polaca en las partes explicativas. La importantísima publicación del diccionario básico polaco-gallego y gallego-polaco (que ya se está elaborando) va a ser la siguiente etapa de la mencionada construcción de las bases cognitivas gallegas en Polonia. Una de las actividades fundamentales del Centro ha sido la creación y el continuo desarrollo de la biblioteca gallega, la que hoy en día cuenta con más de 400 volúmenes de diferentes materias relacionadas con la realidad y la cultura gallegas, diccionarios, manuales y textos canónicos de la literatura de Galicia, así como con una fonoteca y filmoteca básica.

El idioma constituye el instrumento fundamental que posibilita acceder a la cultura ajena, sin embargo para profundizar bien en el idioma y entenderlo rotundamente no hay, sin duda alguna, manera más eficaz que a través de la cultura de sus hablantes. Partiendo de este principio se ofrecen simultáneamente en el Centro cracoviano los cursos de cultura y literatura gallegas, así como de la traducción de literatura. En las aulas de "Cultura e literatura galegas" los estudiantes pueden adquirir el conocimiento de algunos elementos esenciales de la geografía y la historia de Galicia, algunos aspectos antropológicos (como la música, la mitología popular, la gastronomía, las fiestas populares), el cine y la literatura, desde el período de la lírica medieval hasta los tiempos de hoy. Estas aulas gozan de muy buena recepción por parte del alumnado y no sólo el del ambiente de la Filología Románica sino también de otras filologías y a veces de otras carreras humanísticas. Es una de las clases cruciales para la trasmisión de las primeras bases cognitivas, de despertar el interés tanto por las cuestiones particulares (la música, la etnografía, la metafísica popular, el paisaje) como por el contexto histórico y social, el de una cultura históricamente minorizada, agonizante; y cómo no, también por la lengua, su situación y condición particular, sus problemas y desafíos. 
Estudiantes del segundo ciclo de las filologías románicas pueden participar en el aula "Literatura galega contemporánea: tradución e promoción". Es otra de las clases cruciales, la etapa final en el proceso académico de iniciación a la realidad de la lengua y la cultura de Galicia. El objetivo de este curso es dotar a los alumnos de los medios necesarios, facilitarles herramientas con las que puedan escoger un autor, un texto literario, realizar su traducción y finalmente intentar publicarla. Los alumnos que frecuentan esta clase deben poseer ya el conocimiento de la lengua gallega por lo menos en el nivel medio. Y la finalidad última de esta materia es la publicación de las mejores traducciones hechas por los alumnos, como producto final resultado de un esfuerzo común del grupo y del profesor. Es en este curso donde confluyen, según parece, tres de los aspectos más importantes de la influencia de los Centros de Estudos Galegos. Por una parte se enfoca la didáctica y la promoción: dotamos a los alumnos de los conocimientos sobre la lengua y la literatura gallegas, y promovemos la cultura a través de una publicación. Por otra parte se introduce al alumno en el importantísimo campo del interés profesional, apoyando y difundiendo su trabajo.

Gracias a los cursos realizados hasta ahora conseguimos publicar dos números de Almanach galicyjski (Almanaque galego), revista concebida como un espacio de reflexión sobre diferentes aspectos de la cultura gallega. El primer número, que salió en el año 2003, es una antología de textos de carácter introductorio, escritos por los especialistas de las universidades gallegas y traducidos al polaco, sobre la identidad y la realidad cultural gallega, a los que acompaña una pequeña antología de la prosa y poesía contemporánea de Galicia en la traducción al polaco. El segundo número, publicado en el año 2007 y dedicado a Rafael Dieste, novelista y ensayista gallego del siglo $\mathrm{XX}$, incluye una selección de textos de sus libros Dos arquivos de trasno y Historias e invenciones de Félix Muriel vertidos al polaco, junto con ensayos sobre el autor y sobre los textos en cuestión.

Como fruto del "I Encuentro Nacional: lenguas y culturas minoritarias de la Península Ibérica en los estudios polacos”, organizado en Cracovia gracias a la colaboración entre los centros cracoviano 
y varsoviano, conseguimos publicar en el año 2007, bajo el título $P o-$ licromía, un tomo de artículos que giran alrededor de la presencia de las tres lenguas y culturas minoritarias de España (vasca, catalana y gallega), entre los intereses de los especialistas en diferentes esferas del hispanismo en Polonia.

Varias promociones de alumnos han contribuido a la preparación del esqueleto y de los textos para la página web de los Centros de Estudos Galegos de Cracovia y de Varsovia (www.galicia.pl). El portal funciona en dos versiones, en polaco y en gallego, constituyendo un espacio importante de la difusión de la cultura gallega en Polonia. Hasta ahora es la única fuente tan compleja de información sobre Galicia en polaco. La página proporciona información sobre la lengua, literatura, geografía, historia, cultura (gastronomía, leyendas, música, fiestas); enlaces a sitios de interés, a los diccionarios on-line del gallego, actualidad e información sobre los Centros de Estudos Galegos y su oferta educativa en las respectivas universidades polacas. El mérito más destacado de la página web en el aspecto didáctico y de difusión es que sigue siendo este medio modernísimo un espacio casi inagotable, donde los alumnos pueden publicar sus textos de investigación y sus traducciones, lo que constituye un impulso aún más potente no sólo para profundizar en la materia sino para contribuir a la difusión.

Aparte de la actividad didáctica del Centro, uno de los campos más atractivos de su actuación es el de promover la ciencia y la cultura viva de Galicia mediante la organización de simposios y encuentros con los especialistas de diferentes esferas de estudios gallegos, así como de conciertos, monodramas de narradores orales, veladas literarias u otros eventos culturales. Y si la promoción de Galicia a través de sus estudios por razones obvias se limita casi exclusivamente al ambiente académico, la difusión cultural siempre ha intentado salir fuera de las aulas y dar con un auditorio más amplio. Gracias a la subvención anual otorgada por la Xunta de Galicia para este tipo de actividades, conseguimos organizar durante una década de la presencia del Centro en la Universidad Jaguelónica varias conferencias de diferentes temas relacionados con la realidad y la identidad gallega, 
muestras de la literatura y del cine gallegos y numerosos eventos culturales en los que participaron escritores, poetas, actores y músicos gallegos. Desgraciadamente, desde hace tres años, Europa padece una profunda crisis económica que, sabido es de todos, afecta también al Estado Español y dentro de éste a la Región Autonómica de Galicia, lo que ha provocado la reducción notable de la cuota de la subvención, obstaculizando, esperemos de modo pasajero, la intensidad y la calidad de las actividades culturales que se ofrecían hasta ahora. De todas maneras merece la pena mencionar el logro del Centro en esta materia por orden cronológico:

\section{Año 2001}

- Monodrama del cuentacuentos gallego Cándido Pazó.

\section{Año 2002}

- Concierto de Cantigas de Santa María, por un grupo de músicos medievales de Varsovia (Paulina Ceremużyńska: soprano, tar, adufe; Zofia Dowgiałło: arpa; Natalia Babińska: fídula).

- Espectáculo musical Polo camiño de Santiago a cargo de María Xosé López Villar (gaita, zanfonía) y Santi Prego (cuentacuentos).

\section{Año 2003}

- Concierto del grupo folk de Santiago de Compostela Os Estalotes.

- Conferencia de Antón Baamonde, filósofo y ensayista gallego: Galicia hoxe, a reinvención dun país.

\section{Año 2004}

- Concierto del grupo folk Leilía.

- Monodrama del cuentacuentos gallego Kiko Cadaval: Falar por falar.

\section{Año 2005}

- Conferencias ofrecidas por el profesor Arturo Casas de la Universidade de Santiago de Compostela: Rafael Dieste en tres tempos: 
Galiza nos anos 20, Segunda República e exilio y Rumbos da poesía galega actual.

- Velada poética bilingüe Poesía galega na tradución con la participación de Arturo Casas.

- Monodrama del cuentacuentos gallego Carlos Blanco Contando baixiño.

- Conferencia y velada poética: Galicia ibérica - un país do límite Oeste. Galicia onte e hoxe con la Asociación Cultural Willa Decius de Cracovia.

- Concierto de Fran Pérez (guitarra), música gallega alternativa.

\section{Año 2006}

- Visita del poeta y narrador gallego Fran Alonso: conferencia y lectura bilingüe de sus textos.

- Península en verso, velada poética con la participación de Yolanda Castaño (junto con el Instituto Cervantes).

- I Encuentro Nacional: Lenguas y culturas minoritarias de la Península Ibérica en los estudios polacos, organizado para consolidar comunidades científicas interesadas por la temática relacionada con las minorías peninsulares.

- Concierto del grupo folk polaco Suspiria \& Robertos de Lira: música tradicional gallega para voz, zanfonía, flauta, guitarra y pandereta.

- Encuentro de cine gallego. Muestra y discusión: Cortometrajes La buena caligrafía, Rosas.

- Conferencia As linguas da Arte en Galicia, a cargo del prof. Martín Rodríguez Caeiro de la Universidade de Vigo.

- Seminario Aproximación á historia do cine en Galicia, a cargo de la profesora Anxela Ma Nantes Pérez de la Escola Superior de Artes Cinematográficas de Galicia.

\section{Año 2007}

- Monodrama de la cuentacuentos gallega Paula Carballeira Contos que conto para que contes. 
- Monodrama del cuentacuentos gallego Avelino Gónzalez Contrabando de linguas.

\section{Año 2008}

- Conferencia y concierto Os autores galegos da música clásica, a cargo de la pianista gallega Tamara Lorenzo Gabeiras.

- Muestra del cortometraje gallego As cores do frio 2 (5 películas), versiones subtituladas en polaco (las traducciones de los estudiantes de la clase de traducción literaria de gallego).

- Conferencia del profesor Manuel González González de la Universidade de Santiago de Compostela (Secretario de la Real Academia Galega) O galego - unha lingua que busca asegurar o seu futuro.

- Concierto de cantigas de amigo E moirome de amor a cargo del grupo medieval gallego Alienor (Paulina Ceremużyńska: voz; Zofia Dowgiałło: arpa gótica; Fernando Reyes Ferrón: cítola; Carlos Castro: percusión).

\section{Año 2010}

- Concierto del dúo Espido (Guadi Galego, Guillerme Fernández, música del mundo).

\section{Año 2012}

- Conferencia de la poeta y traductora gallega Lucía Rodríguez Caeiro Traducindo á miña propia lingua.

- Espectáculo de la poesía y música gallega en el marco del Krakowski Salon Poezji en el Teatro Słowackiego Życie tutaj to zjawisko atmosferyczne (Joanna Mastalerz, Dominika Bednarczyk, Radosław Krzyżowski [lectura de textos]; Lindsey Davidson [gaita]; Paweł Wajrak [violín]; Michał Nagy [guitarra]). 


\section{Experiencias particulares y perspectivas}

No cabe duda que hoy en día es la preparación previa del mercado la que constituye un factor imprescindible en la difusión eficaz de un espacio cultural totalmente desconocido, y antes de todo afecta esta norma a la literatura, el más universal portavoz de la identidad y conciencia colectiva de una nación. La posición del promotor en este campo resulta ser muy gratificante, ya que permitiendo sacar lo mejor de un terreno virgen y casi inexplorado, proporciona el sentido de misión, por el otro lado sin embargo, supone diferentes tareas suplementarias. Sumamente excepcional resulta ser la situación del promotor de la literatura totalmente ignota. Para actuar con éxito en este terreno en la mayoría de los casos hay que compaginar la voluntad y la ciencia del traductor con el conocimiento profundo de la materia en cuestión para saber planificar estratégicamente y realizar todas las etapas de promoción.

Se puede afirmar que la promoción y la traducción de las literaturas minorizadas y poco conocidas van, sin duda, detrás de la investigación. Y lo que es más, para llevar a cabo con éxito la promoción de una literatura desconocida es imprescindible dejarle vivir su propia vida, independiente en la traducción, porque ésta es la única forma de vivir de un texto literario. Resumiendo, la investigación, la promoción y la traducción se complementan de manera imprescindible, y por eso creo que podemos arriesgar la constatación de que sólo la cooperación y la simultaneidad de los tres factores producen frutos duraderos. El verdadero diálogo intercultural se establece en el mercado libre.

El conocimiento de la cultura gallega en Polonia es hoy relativamente escaso, pero bien estable y creo que con futuro. El interés por las lenguas ibéricas (castellano y portugués) crece en Polonia gradualmente desde hace 20 años. Y a pesar de que suena paradójico, cuanto más interés haya por la lengua y cultura castellana o portuguesa, más interés se inspirará por las lenguas minoritarias peninsulares y más posibilidades habrá de promoción de estas últimas. De esta 
situación hay que sacar el máximo provecho. Frente al nuevo paradigma socio-lingüístico internacional (la Europa de las regiones), la más potente vía de promoción de las culturas minoritarias resulta ser hoy, a mi parecer, la de trasmitir las identidades particulares a través de una visión de la Península Ibérica como un organismo plurilingüe y multicultural.

El objetivo principal del Centro de Estudos Galegos de Cracovia es hacer el máximo esfuerzo para que la cultura gallega se considere, por lo menos en Polonia, parte integral del canon europeo, tal y como merece. 